\title{
Therapeutic strategy in patients with long-lasting essential hypertension with comorbid type 2 diabetes mellitus and obesity
}

\author{
Borys 0. Shelest, Olena V. Hryhorian, Vladyslava V. Sarkis-Ivanova, Yuliia 0. Kovalova, \\ Valeria V. Brek, Oleksiy M. Shelest
}

Kharkiv National Medical University, Ukraine

\begin{abstract}
Background: Presently the level of blood pressure (BP) control is extremely unsatisfactory in hypertensive patients throughout the world. The aim of our study was to find the optimal drug therapy for patients with hard-to-treat essential hypertension (EH) associated with type 2 diabetes mellitus (T2DM) and obesity, namely the comparison of strategies of fixed and non-fixed combination.

Material and methods: Eighty-seven patients with EH, T2DM and obesity were enrolled into the study. Two groups were formed: the $1^{\text {st }}$ group - 41 patients received antihypertensive therapy in the form of unfixed combination of drugs ("multi-pill") perindopril, indapamide and amlodipine; the $2^{\text {nd }}-46$ patients, who received the same drugs, but in a fixed-dose combination ("single pill").

Results: A favorable treatment result was found for fixed-dose combination of antihypertensive drugs, with signifcant reduction in the frequency of visits to the doctor: relative risk (RR) -1.27 (95\% CI: 1.01-1.61), p = 0.045, and odds ratio $(\mathrm{OR})-3.10(95 \% \mathrm{CI}: 1.05-9.13), \mathrm{p}=0.04$. That indicates that patients on fixed-dose combination were less likely to visit a doctor with complaints. Patients on single-pill therapy were less likely to get to progression (worsening) group in contrast to multi-pill non-fixed combination: RR -1.37 (95\% CI: 1.02-1.84), $\mathrm{p}=0.03$; OR - 2.91 (95\% CI: 1.12-7.59), $\mathrm{p}=0.03$.

Conclusion: The single-pill triple combination has significant advantage compared to multi-pill regimen in hard-totreat hypertensive patients with comorbid T2DM and obesity. Fixed-dose triple combination leads to significantly faster achievement of blood pressure control.
\end{abstract}

Key words: single-pill; essential hypertension; comorbidity; treatment 


\section{Introduction}

A high level of blood pressure is among the main reasons of early disabling cardiovascular complications (CVC) such as myocardial infarction and stroke. Poor control of blood pressure (BP) remains among the major problems in contemporary cardiology. At the moment $\mathrm{BP}$ control in hypertensive patients is extremely unsatisfactory [1]. Unsatisfactory national cardiovascular regulations, low patients' adherence to prescribed treatment schemes, and the so-called specialists' inertia are factors responsible for the mentioned problem. Also it is worth to point out the next important factor promoting inappropriate BP control is the confined use of antihypertensive drug combinations. Despite the fact that there is enough evidence that combination treatment leads to more effective BP control in comparison to monotherapy [2].

Nowadays, there are drugs that can effectively affect blood pressure and therefore reduce the risk of stroke, kidney and cardiovascular disease in the arsenal of the clinician, but uncontrolled blood pressure and low adherence to antihypertensive drugs remains one of the main clinical problems [3].

The treatment of essential hypertension $(\mathrm{EH})$ predominantly includes well-known medications from five main classes of drugs: angiotensin-converting enzyme inhibitors (ACEIs), angiotensin II receptor blockers (ARBs), diuretics, calcium channel blockers (CCB), and $\beta$-blockers. There is bounded variation by race, age, gender in choosing of antihypertensive agents. Only $58 \%$ of treated patients had SBP $<140$, $24 \%$ had unsatisfactory controlled BP with SBP $\geq$ 150 , evidencing the effectiveness of treatment needs to be improved [4].

Long-term prospective studies have shown that patients with hypertension did not receive effective monotherapy and needed an average of two or three drugs for adequate control [5].

Despite mentioned data, BP control is poor in majority of countries. Contemporary investigations revealed, regardless the location (country) and irrespective to the high- or low-income economies, just about $40 \%$ of patients with hypertension are treated; of these approximately about $35 \%$ have well controlled blood pressure with BP values less 140/90 $\mathrm{mm} \mathrm{Hg}[6]$.

All mentioned above justifies the search for adequate methods of treatment of patients with $\mathrm{EH}$, especially in the presence of polymorbidity: type 2 diabetes mellitus (T2DM) with obesity. Special difficulties are present when solving the problem of antihypertensive therapy in high-risk patients with additional comorbidities - T2DM with obesity. Thus, if in the general population of patients with $\mathrm{EH}$ effective control is achieved in approximately $15-20 \%$ of such patients, then when combined with T2DM and obesity, this percentage becomes even more unfavorable, even dramatic values, which in turn leads to an increase in adverse events, CVC and reduced life expectancy and earlier disability $[6,7]$.

The question arises, what strategy of drug treatment in such patients is the most optimal? The problem is exacerbated by the fact that the clinician often decides to prescribe antihypertensive therapy to a patient who has additional pathology (T2DM, obesity) and who has suffered from EH for many years and the antihypertensive treatment used so far has been ineffective. Thus, it is not a question of the so-called first line therapy, but of solving the question of finding an effective strategy in such (polymorbid) patients with $\mathrm{EH}$ who have experienced treatment failure and have poorly controlled blood pressure despite prescribed therapy.

The aim of the study was to find the optimal drug therapy for patients with long-lasting essential hypertension associated with type 2 diabetes mellitus and obesity, namely the comparison of strategies of fixed combination of antihypertensive drugs with the strategy of non-fixed combination.

\section{Material and methods}

The 87 patients with $\mathrm{EH}$, type 2 diabetes and obesity were enrolled into the study. Examined subjects were patients admitted to Cardiology Departments of O.I. Meshchaninov City Clinical Hospital of Ambulance and Emergency Care and Kharkiv Regional Hospital who had elevated arterial blood pressure and were over 37 years of age. Two groups of medicament strategy were formed: the $1^{\text {st }}$ group -41 patients with EH, T2DM and obesity, who received antihypertensive therapy in the form of unfixed combination of drugs or "multi-pill" (NFC) - perindopril at a daily dose of $4 \mathrm{mg}$, indapamide (1.25 $\mathrm{mg}$ per day) and amlodipine $(5 \mathrm{mg})$; the $2^{\text {nd }}$ group - 46 patients with EH, T2DM and obesity, who received the same combination of drugs, but in the single pill — a fixed combination. The drug was used in a dose of 1 tablet per day, regardless of food intake, preferably in the morning.

In the study, the combination of EH with T2DM and obesity required the application of the antidiabetic and hypolipidemic therapy in addition to the antihypertensive treatment. Metformin or, if necessary, a combination of metformin with gliclazide, and 
atorvastatin, or rosuvastatin was used. The patients with the stable coronary artery disease were prescribed acetylsalicylic acid. Thus, the treatment strategy was to act on the normalization of blood pressure and blood glucose, lowering total cholesterol and triglycerides, and included diet aimed at weight loss.

Two treatment regimens were evaluated by comparing their positive and negative effects. After that frequencies of good and negative results in the groups were calculated. The scheme with an unfixed combination of antihypertensive drugs was considered as a control to the scheme with a fixed combination. When calculating the relative risk (RR), visiting a physician due to any kind of complaints associated with hypertension-mediated organ damage (headache, chest pain, shortness of breath, fatigue, the appearance of flicker before the eyes, difficulty falling asleep, mood swings, etc.) was referred as negative results.

The both groups were matched by all investigated parameters - age, gender, additional risk factors, physical state etc.

All patients signed informed consent and Institutional Ethical Committee of the participating center approved the protocol of the study (number 2018/05/02), that was performed according to the requirements and norms of the ICH GCP (2002), and following the Declaration of Helsinki, as well as standard provisions on ethics of the Ministry of Health of Ukraine No. 66 13.02.2006.

The examination of hypertensive patients with T2DM and obesity was carried out 3 and 6 months after treatment. The target level of SBP was considered as $130 / 80 \mathrm{~mm} \mathrm{Hg}$.

Statistical analysis. The data is presented as mean \pm standard deviation $(\mathrm{M} \pm \mathrm{SD})$ or percentage $(\%)$. To determine the association between treatment strategy and outcome (result of the therapy) the relative risk and the odds ratio (OR) with $95 \%$ confidence interval (CI) were calculated. OR is most often used in case-control studies, but it can also be used in a onestep intergroup study and in a cohort study (with some assumptions) [8]. The target levels' frequencies and the medicament treatment regimen were compared between two groups by the $\chi^{2}$ test. Statistical significance was considered as $\mathrm{p}$ value $<0.05$. Med Cal (v. 19.3) program was used to calculate and analyze obtained data.

\section{Results}

The baseline characteristics of enrolled patients are presented in Table 1.

The baseline characteristics of enrolled patients show that patients from both treatment regimens

Table 1. Baseline characteristics of patients in the study groups (M $\pm S D$ )

\begin{tabular}{|c|c|c|c|}
\hline Parameters & All patients & $\begin{array}{l}\text { Fixed combination (single-pill) } \\
\qquad(\mathrm{n}=46)\end{array}$ & $\begin{array}{l}\text { Non-fixed combination (multi-pill) } \\
\qquad(\mathrm{n}=41)\end{array}$ \\
\hline Age [yrs] & $59.67 \pm 9.32^{*}$ & $58.87 \pm 8.19^{*}$ & $59.53 \pm 9.51^{*}$ \\
\hline Gender & $\begin{array}{l}\text { Males: } 39(45.4 \%)^{*} \\
\text { Females: } 48(54.6 \%)\end{array}$ & $\begin{array}{l}\text { Males: } 21(45.6 \%)^{*} \\
\text { Females: } 25(54.4 \%)\end{array}$ & $\begin{array}{l}\text { Males: } 18(43.9 \%)^{*} \\
\text { Females: } 23(56.1 \%)\end{array}$ \\
\hline Systolic blood pressure [mm Hg] & $161.32 \pm 7.65^{*}$ & $161.56 \pm 6.98^{*}$ & $160.89 \pm 7.49^{*}$ \\
\hline Diastolic blood pressure [mm Hg] & $96.78 \pm 4.92^{*}$ & $96.89 \pm 4.87^{*}$ & $95.91 \pm 5.01^{*}$ \\
\hline $\mathrm{HbA}_{1 \mathrm{c}}(\%)$ & $7.1 \pm 1.41^{*}$ & $7.0 \pm 1.42^{*}$ & $7.1 \pm 1.33^{*}$ \\
\hline EH duration [yrs] & $11.12 \pm 4.34^{*}$ & $10.91 \pm 4.02^{*}$ & $11.65 \pm 3.97^{*}$ \\
\hline Obesity duration [yrs] & $13.32 \pm 4.43^{*}$ & $14.03 \pm 3.53^{*}$ & $13.11 \pm 4.39^{*}$ \\
\hline T2DM duration [yrs] & $7.83 \pm 3.32^{*}$ & $8.05 \pm 2.81^{*}$ & $7.66 \pm 2.90^{*}$ \\
\hline $\mathrm{BMI}\left[\mathrm{kg} / \mathrm{m}^{2}\right]$ & $35.43 \pm 3.46^{*}$ & $36.11 \pm 3.52^{*}$ & $35.03 \pm 3.43^{*}$ \\
\hline Heart failure [NYHA FC] & $\begin{array}{c}\text { 0: } 5(5.8 \%)^{*} \\
\text { I: } 19(21.8 \%)^{*} \\
\text { II: } 63(72.4 \%)^{*}\end{array}$ & $\begin{array}{c}0: 3(6.5 \%)^{*} \\
\text { I: } 10(21.7 \%)^{*} \\
\text { II: } 33(71.8 \%)^{*}\end{array}$ & $\begin{array}{c}\text { 0: } 2(4.9 \%)^{*} \\
\text { I: } 9(21.9 \%)^{*} \\
\text { II: } 30(73.2 \%)^{*}\end{array}$ \\
\hline Smokers $(\%)$ & $28(32.2 \%)^{*}$ & $15(32.6 \%)^{*}$ & $13(31.7 \%)^{*}$ \\
\hline Dyslipidemia (\%) & $60(69.0 \%)^{*}$ & $31(67.4 \%)^{*}$ & $29(70.7 \%)^{*}$ \\
\hline LV hypertrophy (\%) & $87(100.0 \%)^{*}$ & $46(100.0 \%)^{*}$ & $41(100.0 \%)^{*}$ \\
\hline Carotid intima-media thickness > $0.9 \mathrm{~mm}(\%)$ & $55(63.2 \%)^{*}$ & $29(63.0 \%)^{*}$ & $26(63.5 \%)^{*}$ \\
\hline Microalbuminuria (20 to $200 \mathrm{mg} / \mathrm{L}$ ) (\%) & $71(81.6 \%)^{*}$ & $38(82.6 \%)^{*}$ & $80.5 \%)^{*}$ \\
\hline
\end{tabular}

*the $\mathrm{p}$ level significance $\mathrm{p}>0.1$ (differences between groups); EH — essential hypertension; T2DM — diabetes mellitus type 2; BMI — body mass index; LV — left ventricle; NYHA FC - New York Heart Association Functional Classification 
Table 2. Influence of single-pill (fixed combination) and multi-pill (unfixed combination) strategies on the frequency of visits to the doctor, 6-minute walking test, and clinical state in patients with essential hypertension, type 2 diabetes mellitus (T2DM) and obesity

\begin{tabular}{|l|c|c|}
\hline & RR (95\% CI) & OR (95\% CI) \\
\hline Visiting a physician & $1.27(1.01-1.61)$ & $3.10(1.05-9.13)$ \\
\hline$p$ value & $p=0.045$ & $p=0.04$ \\
\hline 6-minute walking test & $0.99(0.83-1.18)$ & $0.96(0.29-3.11)$ \\
\hline$p$ value & $p=0.94$ & $p=0.94$ \\
\hline Clinical worsening (progressing) & $1.37(1.02-1.84)$ & $2.91(1.12-7.59)$ \\
\hline$p$ value & $p=0.03$ & $p=0.03$ \\
\hline
\end{tabular}

$\mathrm{RR}$ — relative risk; $\mathrm{Cl}$ — confidence interval; $\mathrm{OR}$ — odds rate

were consequently matched by all investigated parameters.

A favorable treatment result was found in the polymorbid group receiving fixed antihypertension combination that was proved by significant reduction in the frequency of visits to the doctor, with calculated RR - 1.27 (95\% CI: 1.01-1.61), $\mathrm{p}=0.045$ and $\mathrm{OR}-3.10(95 \% \mathrm{CI}: 1.05-9.13), \mathrm{p}=0.04$ (Tab. 2).

Thus, this calculation indicates in favor of a scheme with a fixed combination compared to a non-fixed one. Thus, values of relative risk of 1.27 (95\% CI: 1.01-1.61) indicate that patients who received a fixed combination of drugs were less likely to visit a doctor with complaints on hypertensionmediated target organ damage (HMTOD).

The influence of the two treatment options on the dynamics of the clinical condition assessed by the 6-min walking test was also analyzed. Improving the performance of the 6-minute walking test were recorded in $96 \%$ of patients taking a fixed triple combination, and in the group taking three separate drugs; the increase in test scores was observed in $85 \%$ of patients.

Comparison of 6-min walking test results did not showed significant advantage of the triple regimen in one tablet over taking three separate antihypertensive drugs. It was found, that calculations of RR -0.99 (95\% CI: 0.83-1.18), $\mathrm{p}=0.94$ and OR -0.96 (95\% CI: $0.29-3.11), \mathrm{p}=0.94$ indicate that the probability of clinical improvement did not reach statistical significance between groups (Tab. 2).

Progression (worsening) of patients' condition with EH, T2DM mellitus and obesity was assessed by the dynamics of structural and functional parameters of the target organs (heart, kidneys and blood vessels). It was revealed (Tab. 2) that patients taking fixed combination were less likely to get to progression group (group with worsening of condition) than those on non-fixed combination: RR -1.37 (95\%
CI: $1.02-1.84), \mathrm{p}=0.03$; OR $-2.91(95 \% \mathrm{CI}:$ $1.12-7.59), \mathrm{p}=0.03$.

This result of calculations of HR and OR indicates that the use of triple-drug single-pill combination (SPC) is much more effective in preventing deterioration of the target organs in hypertensive patients with polymorbidity. We can assume that this is due to more careful control and faster achievement of target blood pressure levels.

After 3-month treatment (Fig. 1) with a threecomponent antihypertensive regimen the target levels of systolic blood pressure were achieved in 33 hypertensive patients on unfixed combination, and number of patients on fixed combination who reached target levels was $44(\mathrm{p}<0.001)$. An important result is the data of blood pressure control after 6 months, which demonstrate the effectiveness of both selected strategies in achieving the target levels of blood pressure - $97.83 \%$ and $92.68 \%$ of patients receiving fixed and non-fixed combinations, respectively (Fig. 1). Thus, there is a significant advantage of antihypertensive therapy with the use of a fixed combination, which led to a much faster achievement $(\mathrm{p}<0.05)$ of blood pressure $-130 / 80 \mathrm{~mm}$ $\mathrm{Hg}$.

Thus, taking a fixed combination (an ACE inhibitor - perindopril, a diuretic — indapamide and a calcium channel blocker - amlodipine) along with the antihyperglycemic therapy (metformin or a combination of metformin and gliclazide) and recommendations for lifestyle modification was significantly better compared with non-fixed combinations of antihypertensive drugs (Figure 1): after 3 months it lead to significantly higher percentage of patients achieving target blood pressure $-95.65 \%$ (44 patients) against $80.49 \%$ (33 patients) $\left(\chi^{2}=4.84 ; \mathrm{p}=0.03\right)$ and after 6 months $97.83 \%$ ( 45 patients) against $92.68 \%$ (38 patients) $\left(\chi^{2}=1.30\right.$; $\mathrm{p}=0.26$ ). The fixed combination definitely reduces the risk of progression (clinical worsening) of the hypertensive patient with T2DM mellitus and obesity 


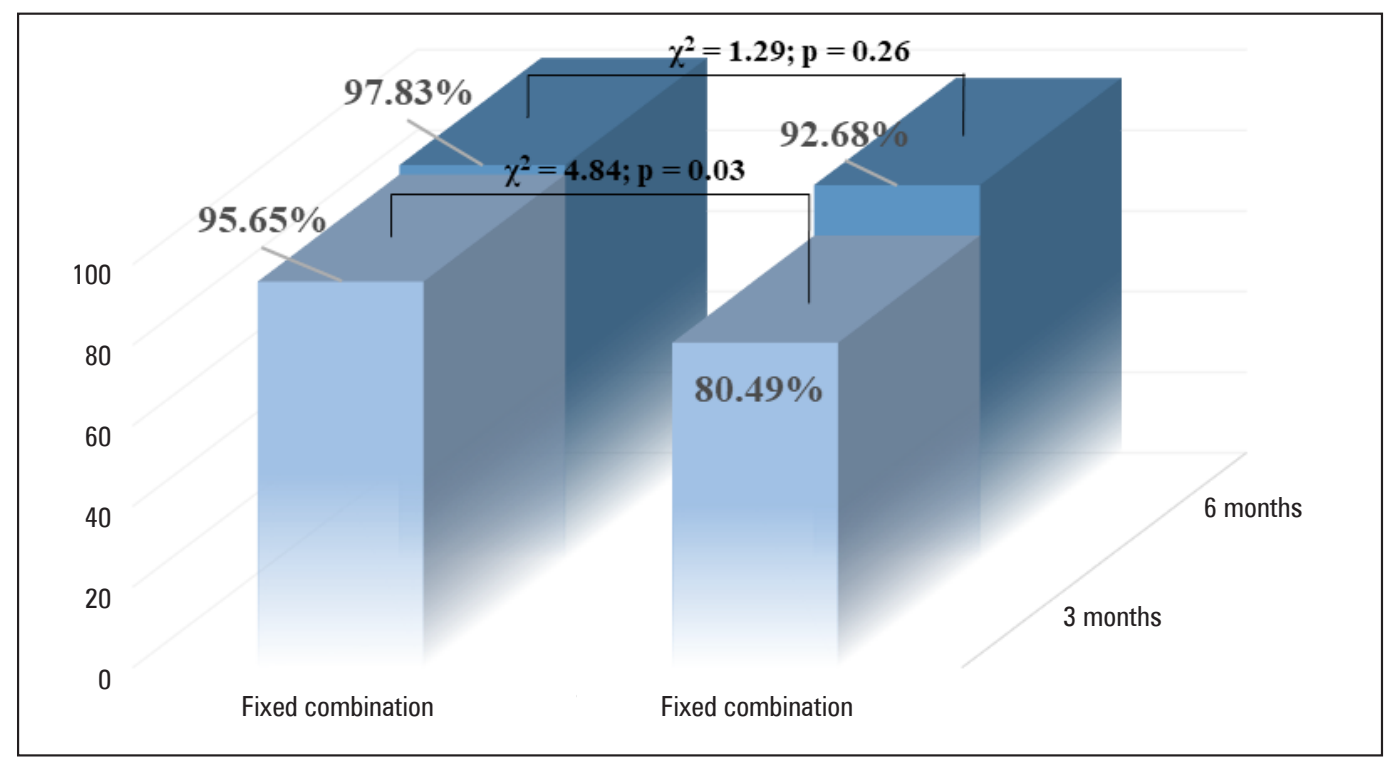

Figure 1. Results of studied treatment strategies: after 3 months and after 6 months. Results are presented as proportions of patients (\%) achieved target blood pressure (BP)

during 6 months of treatment: $80.43 \%$ vs. $58.54 \%$ $\left(\chi^{2}=4.90 ; p=0.03\right)$.

\section{Discussion}

The mentioned triple combination was chosen for the study because of the best evidence based data among the drugs used in hypertension. Perindopril is one of the best representatives of the ACE inhibitor class. It showed high hypotensive efficacy, reduction of overall mortality in patients with $\mathrm{EH}$, as well as a probable reduction in the overall risk of cardiovascular complications in patients with stable coronary heart disease and preserved left ventricular function (ASCOT-BPLA, ADVANCE, HYVET, EUROPA, PERSPECTIVE, PERTINENT). Indapamide is also the optimal drug in its class. It is a metabolically neutral diuretic that has a significant antihypertensive effect and is proven to reduce the incidence of cardiovascular complications in patients with hypertension (HYVET, ADVANCE, PROGRESS, PATS). Amlodipine, in turn, is one of the most powerful $\mathrm{CCB}$. It showed a marked reduction in cardiovascular complications (both cardiac and cerebral) in patients with $\mathrm{EH}$, especially in combination with ACE inhibitors (ASCOT and ACCOMPLISH). In addition, both perindopril and amlodipine have pronounced renoprotective properties $[6,9]$.

It is desirable to underline that the main feature of our study was that medicament treatment in hypertensive patients with high CV risk and with previous history of ineffective BP lowering treatment was investigated. The majority of publications are devoted to the problem of the first-line therapy [10-12]; in contrast, we tried to find out better strategies in those patients who were unsuccessful with recommended first-line treatments. Also we tried to evaluate (to measure) exact preferences of single-pill triple regimen over multi-pill format.

Nowadays the issue of antihypertensive therapy in diabetics remains the cornerstone, and a lot of research has been devoted to this aspect, and often their results are contradictory. In general, it can be concluded from meta-analyses that antihypertensive treatment significantly reduces cardiovascular outcomes in diabetic and non-diabetic patients. Diabetic patients have uncertain benefits in intensive SBP lowering to less $120 \mathrm{~mm} \mathrm{Hg}$, while such intensive $\mathrm{BP}$ control is associated with evident benefits in those without diabetes mellitus [13]. At the same time, reducing ESRD risk has been evidenced only in diabetics (when SBP $<130 \mathrm{~mm} \mathrm{Hg}$ ). Meta-analyses showed that all BP-lowering drugs are effective in diabetic patients, with renin-angiotensin system blockers preferably included in the treatment schemes of patients with diabetes mellitus [9, 14, 15].

On the contrary, Bangalore's study state that in diabetics RAS blockers have no superior effects compared to other antihypertensive medicaments, such as diuretics, calcium channel blockers, or $\beta$-blockers, in diminishing the risk of undesirable cardiovascular and renal events. These resulted in recommendations of the guidelines of the European and American 
societies on management of hypertension to use any antihypertensive drugs in diabetic people but with normal renal function [16].

Grossman et al. assessed evidence supporting different BP targets in diabetics and reviewed the various guidelines on this topic. Moreover, they discussed the different variants available for the BP lowering in diabetics. The authors are more inclined to choose RAAS blockers as the first-line agents among anti-hypertensive drugs. In this study, as in our investigation, calcium channel blocker and/or thiazide were preferable as second line medications in case it was necessary to use more than one drug for the BP control in patients with diabetes. In Grossman et al.'s study it was stated that new anti-diabetic agents could be more effective in antihypertensive treatment and might change the physician's choice in the treatment of T2DM. On the contrast, we used traditional well-recommended drug as metformin in our study. In addition, it is essential to manage all other risk factors in hypertensive patients with T2DM when decreasing BP. Grossman et al. concluded that individual flexible treatment model, reflecting the general modern trends in medicine, proved the need for individually tailored medicine, adapted specifically for the particular anthropological and morphological characteristics of every person [17]. Such result is also supported by our team, but at the same time, we understand that it is necessary to find optimal treatment recommendation for specific cohort of hypertensive patients - with prolonged history of $\mathrm{EH}$ with comorbid T2DM and obesity.

The systemic review by Brunström Mattias et al. indicates that the available data point out the need for a decrease in SBP to more than $140 \mathrm{~mm} \mathrm{Hg}$ in people with T2DM. At the same time, these authors did not find differences in the effectiveness between the main antihypertensive drugs: angiotensin-converting enzyme inhibitors, ARBs, $\beta$-blockers, calcium channel blockers and diuretics in the prevention of general or cardiovascular mortality, as well as coronary artery disease and ESRD. Furthermore, minor additional effects have been found for stroke and heart failure. It is important to note that in this study there were limitations on the inclusion criteria and patients with type 1 diabetes and very elderly patients with T2DM were excluded from the study, which differs from our study [18].

The initial treatment should be started with two drugs or a single-pill combination of drugs proven to reduce cardiovascular events in patients with diabetes. Recommendation of class A insist on poly-drug strategy to control blood pressure targets (but not usage of ACEI with ARB combination), herewith there is no indication on previous treatment history. The initial treatment for diabetic patients depends on the severity of hypertension, but in our study it is defined by previous treatment effectiveness. In diabetic patients with BP over 160/100 mm Hg, initial pharmacological treatment is recommended with two antihypertensive medications, whereas the presence of such additional risk factor as obesity is not discussed deeply [19].

Also, we want to point out the need for wider implementation of single-pill combination in the routine practice, according to hypertension treatment algorithms included in the 2018 ESC guidelines [6]. Combination treatment leads to a faster achievement of BP control in comparison to monotherapy. That is essential in many cases and can be crucial. Herewith, the implementation of the fixed combination approach into everyday clinical practice should be supported by initiatives aimed at resolving physicians' and patients' barriers such as low experience of use, miscellaneous perception of SPC, lack of confidence in the independence of recommendations, patient-physician communication. The concept of first-line antihypertensive therapy needs to be promoted with help of national CME programs and lists of available SPCs, print media and digital materials [20].

Fixed-dose combinations as initial therapy are often needed to achieve fast blood pressure control [21]. On the other hand, European guidelines do not recommend initial triple therapy under any circumstance, but here we firmly underline that only in initial (starting) phase, but not in general. Moreover, we also emphasize in our work more firm and definite usage of fixed triple combination in high-risk hypertensive patients with diabetes and obesity who had prolonged ineffective treatment history.

There are limited investigations of antihypertensive treatment in polymordid patients who have a lot of additional risk factors, which forced us to do presented study.

The distinguishing feature of our work was that the proven benefits of prescribing a fixed triple combination were calculated. It was also emphasized that the effect of a triple fixed combination was noted only in this high-risk group (that is, with multimorbidity) with a documented history of long-term hypertension and ineffective blood pressure control. In other cases - for example, in patients naïve to antihypertensive therapy, it is most likely to start with a fixed two-component combination. 
It is fundamentally important to note that we included patients with both type 2 diabetes and obesity.

It was an obligatory inclusion criterion to have previous experience of ineffective drug therapy, which, from our point of view, represents a huge proportion of hypertensive patients. In addition, these patients are most often people with modest financial resources.

And, most importantly, specific benefits from a fixed combination were calculated - significantly fewer visits to the doctor, decreased numbers of complaints and concerns raised, improved prognosis - reduction of target organ damage and improved quality of blood pressure control.

The presented study had some limitations a small sample and comparison of frequencies in this case can be regarded as the limit of the study. It is also worth noting the relatively short-term of the study. It can be assumed that a longer observation would make it possible to find additional advantages of a fixed triple combination or, on the contrary, to reduce them.

Further research is needed in this cohort of patients, who are at increased risk — with a large sample size and prolonged exposure.

\section{Conclusion}

The non-first line antihypertensive regimen with single-pill triple combination has significant advantage compared to multi-pill (non-fixed) regimen in hard-to-treat hypertensive patients with comorbid 2 type diabetes mellitus and obesity. Such one-pill combination leads to significantly faster achievement of blood pressure control and decreases the chances of worsening of patients' state or/and diminishes chances to disease development.

\section{Conflict of interest}

The authors declare no conflict of interest, financial or otherwise.

\section{References}

1. Unger T, Borghi C, Charchar F, et al. 2020 International Society of Hypertension Global Hypertension Practice Guidelines. Hypertension. 2020; 75(6): 1334-1357, doi: 10.1161/ HYPERTENSIONAHA.120.15026, indexed in Pubmed: 32370572.

2. Mancia G, Rea F, Corrao G, et al. Two-Drug Combinations as First-Step Antihypertensive Treatment. Circ Res. 2019; 124(7):
1113-1123, doi: 10.1161/CIRCRESAHA.118.313294, indexed in Pubmed: 30920930.

3. Peacock E, Krousel-Wood M. Adherence to Antihypertensive Therapy. Med Clin North Am. 2017; 101(1): 229-245, doi: 10.1016/j. mcna.2016.08.005, indexed in Pubmed: 27884232.

4. Shah SJ, Stafford RS. Current Trends of Hypertension Treatment in the United States. Am J Hypertens. 2017; 30(10): 1008-1014, doi: 10.1093/ajh/hpx085, indexed in Pubmed: 28531239.

5. Oparil S, Acelajado MC, Bakris GL, et al. Hypertension. Nat Rev Dis Primers. 2018; 4: 18014, doi: 10.1038/nrdp.2018.14, indexed in Pubmed: 29565029.

6. Williams B, Mancia G, Spiering W, et al. ESC Scientific Document Group. 2018 ESC/ESH Guidelines for the management of arterial hypertension. Eur Heart J. 2018; 39(33): 3021-3104, doi: 10.1093/eurheartj/ehy339, indexed in Pubmed: 30165516.

7. Mills KT, Bundy JD, Kelly TN, et al. Global Disparities of Hypertension Prevalence and Control: A Systematic Analysis of Population-Based Studies From 90 Countries. Circulation. 2016; 134(6): 441-450, doi: 10.1161/CIRCULATIONAHA.115.018912, indexed in Pubmed: 27502908.

8. Stare J, Maucort-Boulch D. Odds Ratio, Hazard Ratio and Relative Risk. Metodoloski Zvezki. 2016; 13(1): 59-67.

9. Soranna D, Zambon A, Corrao G, et al. Different effects of antihypertensive treatment on office and ambulatory blood pressure: a meta-analysis. J Hypertens. 2019; 37(3): 467-475, doi: 10.1097/HJH.0000000000001914, indexed in Pubmed: 30234773.

10. 2017ACC/AHA/AAPA/ABC/ACPM/AGS/APhA/ASH/ASPC/ NMA/PCNA Guideline for the Prevention, Detection, Evaluation, and Management of High Blood Pressure in Adults: A Report of the American College of Cardiology/American Heart Association Task Force on Clinical Practice Guidelines. J Am Coll Cardiol. 2018; 71: e127-e248.

11. Wright JM, Musini VM, Gill R. First-line drugs for hypertension. Cochrane Database Syst Rev. 2018; 4: CD001841, doi: 10.1002/14651858.CD001841.pub3, indexed in Pubmed: 29667175.

12. Chen YuJ, Li LJ, Tang WLu, et al. First-line drugs inhibiting the renin angiotensin system versus other first-line antihypertensive drug classes for hypertension. Cochrane Database Syst Rev. 2018; 11: CD008170, doi: 10.1002/14651858.CD008170.pub3, indexed in Pubmed: 30480768.

13. Volpe M, Gallo G. Systolic blood pressure target less than $120 \mathrm{mmHg}$ : the 'Chariot Allegory' in hypertension? J Hypertens. 2020; 38(8): 1462-1463, doi: $10.1097 /$ HJH.0000000000002458, indexed in Pubmed: 32687272.

14. Thomopoulos C, Parati G, Zanchetti A. Effects of blood-pressurelowering treatment on outcome incidence in hypertension: 10 - Should blood pressure management differ in hypertensive patients with and without diabetes mellitus? Overview and meta-analyses of randomized trials. J Hypertens. 2017; 35(5): 922-944, doi: 10.1097/HJH.000000000001276, indexed in Pubmed: 28141660.

15. Thomopoulos C, Parati G, Zanchetti A, et al. Effects of blood pressure lowering on outcome incidence in hypertension. 1. Overview, meta-analyses, and meta-regression analyses of randomized trials. J Hypertens. 2014; 32(12): 2285-2295, doi: 10.1097/HJH.0000000000000378, indexed in Pubmed: 25255397.

16. Bangalore S, Fakheri R, Toklu B, et al. Diabetes mellitus as a compelling indication for use of renin angiotensin system blockers: systematic review and meta-analysis of randomized trials. BMJ. 2016; 352: i438, doi: 10.1136/bmj.i438, indexed in Pubmed: 26868137. 
17. Grossman A, Grossman E. Blood pressure control in type 2 diabetic patients. Cardiovasc Diabetol. 2017; 16(1): 3, doi: 10.1186/ s12933-016-0485-3, indexed in Pubmed: 28056987.

18. Brunström M, Eliasson M, Nilsson PM, et al. Blood pressure treatment levels and choice of antihypertensive agent in people with diabetes mellitus: an overview of systematic reviews. J Hypertens. 2017; 35(3): 453-462, doi: 10.1097/HJH.0000000000001183, indexed in Pubmed: 27870655.

19. de Boer IH, Bangalore S, Benetos A, et al. Diabetes and Hypertension: A Position Statement by the American Diabetes Association.
Diabetes Care. 2017; 40(9): 1273-1284, doi: 10.2337/dci170026, indexed in Pubmed: 28830958.

20. Campana E, Cunha V, Glaveckaite S, et al. The use of single-pill combinations as first-line treatment for hypertension: translating guidelines into clinical practice. J Hypertens. 2020; 38(12): 2369-2377, doi: 10.1097/HJH.0000000000002598, indexed in Pubmed: 32833920.

21. Volpe M, Gallo G, Tocci G. Is early and fast blood pressure control important in hypertension management? Int J Cardiol. 2018; 254: 328-332, doi: 10.1016/j.ijcard.2017.12.026, indexed in Pubmed: 29273242. 